\title{
ASYMPTOTIC ANALYSIS OF VERTICAL GEOTHERMAL BOREHOLES IN THE LIMIT OF SLOWLY VARYING HEAT INJECTION RATES*
}

\author{
MIGUEL HERMANNS ${ }^{\dagger}$ AND JOSÉ MIGUEL PÉREZ ${ }^{\dagger}$
}

\begin{abstract}
Theoretical models for the thermal response of vertical geothermal boreholes often assume that the characteristic time of variation of the heat injection rate is much larger than the characteristic diffusion time across the borehole. In this case, heat transfer inside the borehole and in its immediate surroundings is quasi-steady in the first approximation, while unsteady effects enter only in the far field. Previous studies have exploited this disparity of time scales, incorporating approximate matching conditions to couple the near-borehole region with the outer unsteady temperature field. In the present work matched asymptotic expansion techniques are used to analyze the heat transfer problem, delivering a rigorous derivation of the true matching condition between the two regions and of the correct definition of the network of thermal resistances that represents the quasi-steady solution near the borehole. Additionally, an apparent temperature due to the unsteady far field is identified that needs to be taken into account by the near-borehole region for the correct computation of the heat injection rate. This temperature differs from the usual mean borehole temperature employed in the literature.
\end{abstract}

Key words. geothermal heat exchangers, geothermal boreholes, borehole thermal resistances, heat equation, asymptotic matching, intermediate scale

AMS subject classifications. 00A74, 35C20, 35K05, 76M55, 65M05

DOI. $10.1137 / 130930170$

1. Introduction. The continuously increasing cost of energy combined with the growing need for a more sustainable development of mankind is nowadays pushing at all levels a more efficient use of available energy and natural resources. An area of high impact, and therefore of potentially big savings, is the heating and cooling of buildings, which accounts for more than $25 \%$ of world energy consumption [17]. Therefore, sustainable construction and buildings represent a fundamental tool to achieve the sought overall increase in energy efficiency.

Among the different renewable energy sources that can be exploited for the heating and cooling of buildings, low temperature geothermal energy is considered one of the most efficient and promising ones. Geothermal heat pumps, or ground source heat pumps, are conventional heat pumps connected to a ground heat exchanger comprised of vertical boreholes in which a liquid flows through a series of U-shaped pipes and exchanges heat with the surrounding ground. These HVAC (heating, ventilation, and air conditioning) systems can, however, be considered truly renewable only if the ground heat exchanger and the strategy for heat injection and extraction from the ground are properly designed. Otherwise, the ground is progressively heated up or cooled down and thereby thermally exhausted. Therefore, it is critical to be able to correctly and accurately predict the thermal response of the borehole and its surrounding ground during the design stage of the system.

The obvious choice would be to numerically solve the complete three-dimensional

* Received by the editors July 22, 2013; accepted for publication (in revised form) October 10, 2013; published electronically January 28, 2014. This work was supported by project IPT-2011-0877920000 of the Spanish Ministerio de Economia y Competitividad.

http://www.siam.org/journals/siap/74-1/93017.html

†E.T.S.I. Aeronáuticos, Dpto de Motopropulsión y Termofluidodinámica, Universidad Politécnica de Madrid, 28040 Madrid, Spain (miguel.hermanns@upm.es, jomipp@torroja.dmt.upm.es). 
unsteady heat transfer problem that arises from the heat exchange between the ground heat exchanger and the surrounding ground. This is nowadays possible in reasonable amounts of time, thanks to the greatly increased computational capabilities of modern computers $[8,22,23,25]$. However, at the design stage of the HVAC system of a building, parametric studies are used to find the best design, which requires the study of tens if not hundreds of different configurations. In this case, the computational cost of solving the complete problem numerically becomes prohibitive, and more computationally efficient, but still accurate, methods and models are required.

The unsteady thermal problem under consideration presents three intrinsic characteristic times. First is the residence time of the liquid in the pipes, given by $t_{r} \sim H / V$, with $H$ the length of the vertical borehole and $V$ the mean flow velocity of the liquid in the pipes. Second is the characteristic thermal diffusion time in the transverse direction to the borehole, given by $t_{b} \sim r_{b}^{2} / \alpha$, with $r_{b}$ the borehole radius and $\alpha$ the thermal diffusivity of the ground. And third is the characteristic diffusion time along the borehole, given by $t_{H} \sim H^{2} / \alpha$. Due to the extreme slenderness of commonly used vertical boreholes and the requirement of a turbulent flow regime inside the pipes, which imposes a minimum value for the flow velocity, the above introduced characteristic times satisfy the following relation: $t_{r} \ll t_{b} \ll t_{H}$.

A common strategy for developing computationally less expensive, but still accurate, methods and models is to exploit this difference in time scales by concentrating on heat injection or extraction rates that vary with a characteristic time $t_{q}$ that is slow compared to the characteristic transverse diffusion time, $t_{b} \ll t_{q}$. In this case, the flow inside the pipes as well as the thermal conduction problem in the proximities of the borehole can as a first approximation be considered quasi-steady, thus significantly reducing the complexity of the problem to be solved. For example, the complete effect onto the global heat transfer problem of the complex geometry and the thermal properties of the borehole, its grout filling, and its pipes can be condensed into a network of thermal resistances that relates temperatures with heat rates and that can be computed a priori with little computational effort.

To obtain that network of thermal resistances, the quasi-steady heat transfer problem inside and around the geothermal borehole must be solved for a certain set of operating conditions. This requires the numerical $[24,31]$ or analytical $[3,9,10]$ solution of the steady-state heat conduction equation together with the proper set of boundary conditions. While the boundary conditions to be imposed at the outer surface of the U-shaped pipes as well as at the interface between the borehole and the surrounding ground are clear, the one to be imposed far from the borehole, and that links with the outer unsteady heat transfer problem, is less straightforward. Several approaches can be found in the literature for that outer boundary condition.

One approach was initiated by Bennet and coworkers in 1987 [3, 9]. They proposed a mixed-type boundary condition at a certain distance $r_{c}$ from the borehole center, allowing thereby for the inclusion of possible thermal contact resistances at that location. However, neither the distance $r_{c}$ nor the mixed-type boundary condition itself were motivated by the underlying physical problem. Therefore, the dependence of the thermal resistances on it did not make sense. Later on, Hellström [16] continued using the same boundary condition at $r_{c}$ but introduced an alternative definition of thermal resistance. By introducing the mean azimuthal temperature $T_{b}$ at the borehole wall, and using it to define the thermal resistances, he formally eliminated the dependence on $r_{c}$ and on the associated mixed-type boundary condition. However, the numerical values were still varying with $r_{c}$, although very weakly. It has not been until very recently that a completely $r_{c}$-free formulation of the thermal resistances has 
been presented. Claesson and Hellström recently [10] reformulated the problem and its solution procedure in order to be able to directly impose $T_{b}$ as the mean azimuthal temperature at the borehole wall instead of doing it indirectly through a boundary condition located at $r=r_{c}$. As will be shown later, this latter approach leads to the physically and mathematically correct definition of thermal resistances.

Another approach started with the introduction by Hellström of the mean azimuthal temperature at the borehole wall, which spawned the wrong impression that the correct way of computing thermal resistances was by imposing that temperature directly as a Dirichlet boundary condition at the borehole wall [24, 31]. Since there is no physical reason for imposing a uniform temperature there, this approach leads to significant errors, especially when the U-shaped pipes are located close to the borehole wall, as shown in [22] and in the present work.

Finally, a third approach can be found in [22], where unsteady thermal simulations of the borehole and its surroundings are performed and the values of the thermal resistances derived from the long term evolution of those simulations. Although this approach leads to the correct results, it is time-consuming and therefore not really suited for industrial applications. Additionally, the numerical values are dependent on the final time of the performed simulation. However, that dependence will be weak if the final times of simulation are sufficiently large compared to the characteristic transverse diffusion time $t_{b}$.

Although the first of the three described approaches has finally led to the correct definition of thermal resistances, a formally correct derivation of the outer boundary condition has not been presented so far. This requires careful analysis of the asymptotic matching of the steady-state solution close to the borehole with the unsteady heat transfer problem far from the borehole. Therefore, in the present work the thermal response of vertical geothermal boreholes in the limit of slowly varying heat injection rates is studied using matched asymptotic expansion techniques [21]. Of special interest is the zeroth order asymptotic matching between the region near the borehole (inner region) and the region far from the borehole (outer region), which delivers the correct outer boundary condition to be used in the computation of the network of thermal resistances that represents the quasi-steady solution to the inner region. Additionally, the asymptotic matching also delivers an apparent temperature imposed by the outer region onto the inner region that has to be used for the correct computation of the heat injection rate. This temperature differs from that usually employed in the literature.

The work is organized as follows. Section 2 presents the mathematical formulation of the unsteady heat transfer problem inside and around the vertical borehole. The problem is then solved in the limit of slowly varying heat injection rates in section 3 using matched asymptotic expansion techniques. From the obtained results, section 4 derives the network of thermal resistances that describes the quasi-steady thermal response of the borehole vicinity. Thereafter, a theoretical discussion on the existing models for the thermal resistances is carried out in section 5 , followed by a quantitative analysis of the accuracy of these models in section 6 . Finally, concluding remarks are presented in section 7 .

2. Formulation of the thermal problem. Commonly used geothermal boreholes present lengths which are on the order of hundred meters, while their diameters are on the order of tens of centimeters. Taking into account that the thermal diffusivity of the ground $\alpha$ is typically around $10^{-6} \mathrm{~m}^{2} / \mathrm{s}[6,14,29]$, transverse $t_{b}$ and longitudinal $t_{H}$ characteristic diffusion times on the order of hours and centuries are 
respectively obtained. The present work focuses on those operating conditions in which the heat injection rate into the borehole varies with a characteristic time $t_{q}$ that is much larger than $t_{b}$ but much smaller than $t_{H}: t_{b} \ll t_{q} \ll t_{H}$. This asymptotic limit is of practical relevance for the study of the thermal response of geothermal boreholes to loads that vary over periods of a few days up to a few years, the lower time limit being imposed by the condition $t_{b} \ll t_{q}$ and the upper time limit by the condition $t_{q} \ll t_{H}$.

From the point of view of the formulation of the thermal problem, the condition $t_{q} \ll t_{H}$ implies that the thermal diffusion along the borehole can as a first approximation be neglected when compared to the transverse thermal diffusion. This allows the unsteady thermal problem to be formulated in independent two-dimensional planes perpendicular to the borehole, which are only coupled to each other through the fluid temperatures that vary along the pipes inside the borehole.

Consider one of those two-dimensional planes shown in Figure 1. The represented configuration is comprised of two pipes located respectively at $\vec{x}_{1}$ and $\vec{x}_{2}$ inside the borehole of radius $r_{b}$ and whose outer radii are respectively $r_{1}$ and $r_{2}$. Although the shown configuration has two pipes, the results and conclusions derived in the present work are also applicable to any other borehole configuration with a different arrangement of pipes, like double or triple U-pipes, coaxial pipes, etc.

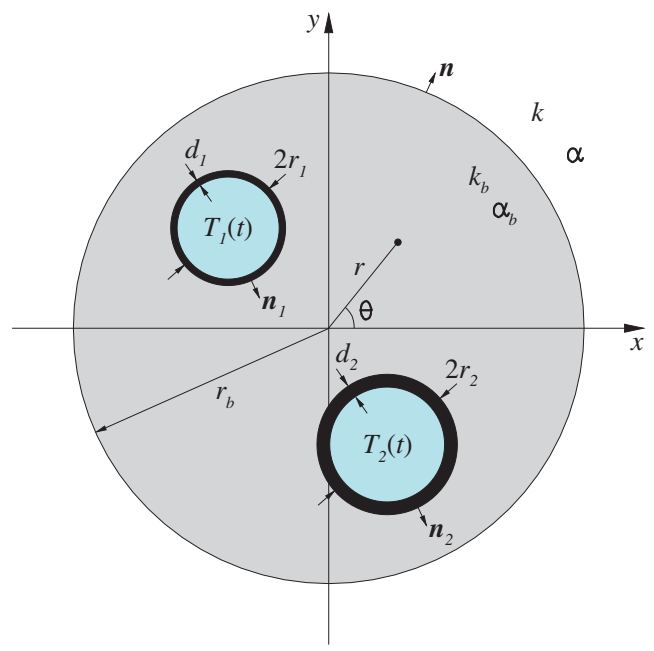

FIG. 1. Cross section of a typical geothermal borehole of radius $r_{b}$ with a single $U$-shaped pipe in it and filled with a grout material with thermal conductivity $k_{b}$ and thermal diffusivity $\alpha_{b}$ that in general differ from the corresponding values $k$ and $\alpha$ of the surrounding ground.

A liquid flows inside the pipes at sufficiently high Reynolds numbers to ensure a turbulent flow regime. This allows the thermal characterization of the liquid to be done through a uniform mean temperature $T_{i}(t)$ at each cross section of each pipe $i[26]$. The heat exchange between the liquid and the pipe walls is governed by the forced convection imposed by the flow and can be characterized by a convective heat transfer coefficient $h_{i}$, different for each pipe, that depends in nonlinear ways on the flow conditions inside the pipe as well as on the transport properties of the liquid [26].

The condition that the heat flux across each pipe wall must be conserved leads to the following boundary condition to be imposed at the outer face of the wall of each 
pipe $i$ :

$$
-k_{b} r_{i} \frac{\partial T}{\partial n_{i}}=\frac{T_{i}(t)-T}{R_{p i}},
$$

where $k_{b}$ is the thermal conductivity of the grout material filling the borehole, $n_{i}$ is the outward-pointing normal direction to pipe $i$, and $R_{p i}$ is the inner thermal resistance of pipe $i$. The inner thermal resistance takes into account the convective heat transfer coefficient $h_{i}$ associated with the flow inside the pipe as well as the thermal resistance associated with the finite thickness $d_{i}$ and thermal conductivity $k_{i}$ of the pipe wall:

$$
R_{p i}=\frac{1}{\left(r_{i}-d_{i}\right) h_{i}}+\frac{1}{k_{i}} \ln \left(\frac{r_{i}}{r_{i}-d_{i}}\right) .
$$

Implicit in the above expressions is the assumption that the heat conduction across the pipe wall can be considered quasi-steady. This is a reasonable approximation taking into account that the characteristic diffusion time across the pipe wall, given by $d_{i}^{2} / \alpha_{i}$, with $\alpha_{i}$ the thermal diffusivity of the pipe wall material, is usually small compared to the characteristic transverse diffusion time of the borehole $t_{b} \sim r_{c}^{2} / \alpha$.

The heat transfer inside the grout material is governed by the unsteady heat conduction equation, which for the case of cylindrical coordinates is given by

$$
\frac{\partial T}{\partial t}=\alpha_{b}\left[\frac{1}{r} \frac{\partial}{\partial r}\left(r \frac{\partial T}{\partial r}\right)+\frac{1}{r^{2}} \frac{\partial^{2} T}{\partial \theta^{2}}\right],
$$

where $\alpha_{b}$ is the thermal diffusivity of the grout material, $r$ the radial coordinate measured from the center of the borehole, and $\theta$ the azimuthal angle. Analogously, the heat transfer inside the ground surrounding the borehole is also governed by the same unsteady heat conduction equation, except that the thermal diffusivity to be employed is that corresponding to the ground, $\alpha$ :

$$
\frac{\partial T}{\partial t}=\alpha\left[\frac{1}{r} \frac{\partial}{\partial r}\left(r \frac{\partial T}{\partial r}\right)+\frac{1}{r^{2}} \frac{\partial^{2} T}{\partial \theta^{2}}\right] .
$$

Across the borehole wall, located at $r=r_{b}$, temperatures and normal heat fluxes must be continuous:

$$
\left.T\right|_{b}=\left.T\right|_{g}, \quad-\left.k_{b} \frac{\partial T}{\partial n}\right|_{b}=-\left.k \frac{\partial T}{\partial n}\right|_{g},
$$

with $k$ being the thermal conductivity of the ground and $n$ the outward-pointing direction normal to the borehole wall.

Far from the borehole, the temperature of the ground must be equal to the unperturbed ground temperature $T_{\infty}$ :

$$
|\vec{x}| \rightarrow \infty: T \rightarrow T_{\infty} .
$$

And finally, the initial condition states that the ground as well as the grout material are initially at the unperturbed ground temperature:

$$
t=0: T=T_{\infty}
$$

For the sake of simplicity, and without altering the applicability of the forthcoming asymptotic analysis to more complex cases, all the parameters of the problem are 
considered to be constant except for the temperatures $T_{i}(t)$ of the liquid flowing inside the pipes. These are assumed to vary in prescribed ways and with a characteristic time of variation that coincides with the characteristic time of variation $t_{q}$ of the heat injection rate. Additionally, and also for the sake of simplicity in the forthcoming development, the thermal diffusivities of the grout material and the ground are considered to be of the same order, $\alpha_{b} \sim \alpha$, an assumption that is reasonable in view of their typical values $[33,34,35]$.

3. Limit of slowly varying heat injection rates. Once the thermal problem has been formulated taking into account that $t_{q} \ll t_{H}$, the next step is to account for the condition that the heat injection rate varies slowly when compared with the characteristic transverse diffusion time, $t_{b} \ll t_{q}$. This is accomplished by carrying out the asymptotic analysis of the formulated thermal problem for the small parameter $\epsilon=r_{b} / \sqrt{\alpha / t_{q}} \sim \sqrt{t_{b} / t_{q}} \ll 1$. This gives rise to two distinct regions in the problem: a quasi-steady inner one at distances $r \sim r_{b}$ from the borehole and an unsteady outer one at distances $r \sim \sqrt{\alpha t_{q}} \gg r_{b}$ from the borehole.

Formally, the asymptotic expansions that will be used are the following:

$$
\begin{aligned}
& \text { inner region: } \Theta_{\text {in }}=\Theta_{\text {in } 0}(R, \theta, \tau)+\sigma_{\text {in }}(\epsilon) \Theta_{\text {in } 1}(R, \theta, \tau)+\cdots, \\
& \text { outer region: } \Theta_{\text {out }}=\Theta_{\text {out } 0}(\eta, \theta, \tau)+\sigma_{\text {out }}(\epsilon) \Theta_{\text {out } 1}(\eta, \theta, \tau)+\cdots,
\end{aligned}
$$

where $\sigma_{\text {in }}(\epsilon) \ll 1$ and $\sigma_{\text {out }}(\epsilon) \ll 1$ have to be obtained as part of the matching process between the two regions and where the following nondimensionalization has been employed:

$$
\Theta=\frac{T-T_{\infty}}{q_{c} / k}, \quad R=\frac{r}{r_{b}}, \quad \eta=R \epsilon=\frac{r}{\sqrt{\alpha t_{q}}}, \quad \tau=\frac{t}{t_{q}} .
$$

The heat injection rate per unit borehole length, whose characteristic value $q_{c}$ appears in the nondimensionalization, will be introduced later on in this section.

Only the zeroth order term of the asymptotic expansion will be obtained here. Therefore, the subscript 0 will be omitted from now on to simplify the notation. Additionally, the presentation will depart from the classical formalism used in asymptotic analysis in order to simplify it and to allow the placing of more emphasis on the physical aspects of the underlying problem than on the mathematical details of the asymptotic analysis.

3.1. Zeroth order inner solution. The unsteady term in the heat conduction equation for the borehole (2.3) and for the ground (2.4) is of order $\Delta_{c} T / t_{q}$, where $\Delta_{c} T$ is the characteristic variation of the temperature in the problem, while the diffusion terms of both equations are of order $\alpha \Delta_{c} T / r_{b}^{2}$. By comparison of one estimation with the other it can be seen that as a first approximation the unsteady terms are negligible due to the condition $t_{b} / t_{q} \ll 1$, and the thermal problem to be solved is comprised of the steady-state heat conduction equation in the borehole and in the ground,

$$
0=\frac{1}{r} \frac{\partial}{\partial r}\left(r \frac{\partial T}{\partial r}\right)+\frac{1}{r^{2}} \frac{\partial^{2} T}{\partial \theta^{2}}
$$

together with the continuity conditions for temperatures and normal heat fluxes at the borehole wall (2.5), the boundary condition at the outer pipe walls (2.1), and the boundary condition at infinity (2.6). Since the fluid temperatures still vary with time, 
the thermal problem to be solved is quasi-steady, and time plays only the role of an additional parameter.

It is well known that the above problem has in general no solution except for certain very particular cases, like the trivial one in which $T_{i}(t)=T_{\infty}$ [7]. The reason for this is that at distances to the borehole of the order of $r \sim \sqrt{\alpha t_{q}} \gg r_{b}$, the unsteady term and the diffusion terms in the unsteady heat conduction equation (2.4) are equally important, rendering the quasi-steady approximation no longer valid in that outer region.

3.2. Zeroth order outer solution. To analyze that outer region the formulated thermal problem is nondimensionalized using the variables introduced in (3.1), leaving the unsteady heat conduction equation in the ground as follows:

$$
\frac{\partial \Theta}{\partial \tau}=\frac{1}{\eta} \frac{\partial}{\partial \eta}\left(\eta \frac{\partial \Theta}{\partial \eta}\right)+\frac{1}{\eta^{2}} \frac{\partial^{2} \Theta}{\partial \theta^{2}} .
$$

This equation has to be solved together with the following initial condition and boundary condition at infinity:

$$
\begin{aligned}
& \tau=0: \Theta=0, \\
& \eta \rightarrow \infty: \Theta \rightarrow 0 .
\end{aligned}
$$

In contrast, the boundary conditions on the pipe walls as well as the continuity conditions across the borehole wall are all substituted by asymptotic matching conditions at $\eta \ll 1$ with the quasi-steady inner region discussed in the previous section.

The asymptotic matching between the inner and outer solutions has two steps. In the first one, a matching of the heat injection rate per unit borehole length $q(\tau)$ between both solutions is imposed. From the perspective of the inner and outer solutions, this means that

$$
\lim _{r \rightarrow \infty} \int_{0}^{2 \pi}-k \frac{\partial T}{\partial r} r \mathrm{~d} \theta=\lim _{\eta \rightarrow 0} \int_{0}^{2 \pi}-q_{c} \frac{\partial \Theta}{\partial \eta} \eta \mathrm{d} \theta .
$$

In the zeroth order approximation to the outer solution, the azimuthal variations of the inner solution are not relevant. Therefore, the outer solution will as a first approximation be axisymmetric, allowing the corresponding matching condition to be rewritten as follows:

$$
\eta \ll 1: \frac{q(\tau)}{q_{c}}=-2 \pi \eta \frac{\partial \Theta}{\partial \eta} .
$$

The value of $q(\tau)$ is a priori unknown and will be obtained from the asymptotic matching of the outer and inner regions. The resulting thermal problem to be solved in the outer region corresponds to an infinite medium with a localized line source of heat of nondimensional intensity per unit borehole length equal to $\bar{q}(\tau)=q(\tau) / q_{c}$. The solution to this problem is well known [7]:

$$
\Theta(\eta, \tau)=\frac{1}{4 \pi} \int_{0}^{\tau} \frac{\bar{q}(\lambda)}{\tau-\lambda} \exp \left[-\frac{\eta^{2}}{4(\tau-\lambda)}\right] \mathrm{d} \lambda
$$

3.3. Asymptotic matching. To complete the asymptotic matching between the outer and inner solutions, the asymptotic behavior of the outer solution when 
$\eta \ll 1$ is required. To accomplish this, the outer solution given in (3.7) is rewritten as follows:

$$
\begin{aligned}
\Theta(\eta, \tau) & =\frac{1}{4 \pi} \int_{0}^{\tau} \frac{\bar{q}(\tau)}{\tau-\lambda} \exp \left[-\frac{\eta^{2}}{4(\tau-\lambda)}\right] \mathrm{d} \lambda \\
& -\frac{1}{4 \pi} \int_{0}^{\tau} \frac{\bar{q}(\tau)-\bar{q}(\lambda)}{\tau-\lambda} \mathrm{d} \lambda \\
& +\frac{1}{4 \pi} \int_{0}^{\tau} \frac{\bar{q}(\tau)-\bar{q}(\lambda)}{\tau-\lambda}\left[1-\exp \left[-\frac{\eta^{2}}{4(\tau-\lambda)}\right]\right] \mathrm{d} \lambda,
\end{aligned}
$$

where $\bar{q}(\tau)$ is added to and subtracted from $\bar{q}(\lambda)$ and where the complete second integral is added to and subtracted from the outer solution. The first of the integrals has a primitive function, which is the exponential integral function:

$$
\frac{1}{4 \pi} \int_{0}^{\tau} \frac{\bar{q}(\tau)}{\tau-\lambda} \exp \left[-\frac{\eta^{2}}{4(\tau-\lambda)}\right] \mathrm{d} \lambda=\frac{\bar{q}(\tau)}{4 \pi} \mathrm{E}_{1}\left(\frac{\eta^{2}}{4 \tau}\right) .
$$

The second one is well defined for the specified integration limits, as the integrand tends to zero or at most to a constant when $\lambda$ approaches $\tau$. Only the third integral requires a more detailed analysis.

Close to the borehole, where $\eta \ll 1$, the integrand of the third integral is zero everywhere except when $(\tau-\lambda) \sim \eta^{2} \ll 1$. Hence, an intermediate asymptotic matching scale $\delta$ is introduced that also tends to zero and additionally satisfies the relation $\eta^{2} \ll \delta \ll 1$. With it, the third integral can be simplified as follows:

$$
\begin{aligned}
I_{3} & =\frac{1}{4 \pi} \int_{0}^{\tau} \frac{\bar{q}(\tau)-\bar{q}(\lambda)}{\tau-\lambda}\left[1-\exp \left[-\frac{\eta^{2}}{4(\tau-\lambda)}\right]\right] \mathrm{d} \lambda \\
& \left.\simeq \frac{1}{4 \pi} \frac{d \bar{q}}{d \tau}\right|_{\tau} \int_{\tau-\delta}^{\tau}\left[1-\exp \left[-\frac{\eta^{2}}{4(\tau-\lambda)}\right]\right] \mathrm{d} \lambda,
\end{aligned}
$$

where the heat injection rate per unit borehole length is expanded in a Taylor series around time $\tau$, taking advantage of the fact that $\tau-\lambda \sim \delta \ll 1$. The remaining integral in (3.10) can be bounded, as its integrand is at most equal to 1 , and therefore $I_{3} \sim$ $\Delta_{c} \bar{q} \delta$, where $\Delta_{c} \bar{q}$ is the characteristic variation experienced by the nondimensional heat injection rate per unit borehole length. This integral turns out to be negligible when compared to the second integral, whose order of magnitude is $\Delta_{c} \bar{q}$. Therefore,

$$
\left.\Theta(\eta, \tau)\right|_{\eta \ll 1}=\frac{\bar{q}(\tau)}{4 \pi} \mathrm{E}_{1}\left(\frac{\eta^{2}}{4 \tau}\right)-\frac{1}{4 \pi} \int_{0}^{\tau} \frac{\bar{q}(\tau)-\bar{q}(\lambda)}{\tau-\lambda} \mathrm{d} \lambda .
$$

Expanding the exponential integral function for small values of its argument, the following final result is obtained:

$$
\left.\Theta(\eta, \tau)\right|_{\eta \ll 1}=-\frac{\bar{q}(\tau)}{4 \pi}\left[\ln \left(\frac{\eta^{2}}{4 \tau}\right)+\gamma\right]-\frac{1}{4 \pi} \int_{0}^{\tau} \frac{\bar{q}(\tau)-\bar{q}(\lambda)}{\tau-\lambda} \mathrm{d} \lambda,
$$

where $\gamma=0.57721 \ldots$ is Euler's constant. To perform the matching with the inner solution, the nondimensionalization is reverted and the logarithm expanded, leading to

$$
\left.T(r, t)\right|_{r \gg r_{b}}=-\frac{q(t)}{2 \pi k} \ln \left(\frac{r}{r_{b}}\right)+T_{a}(q, t)
$$


where the apparent temperature $T_{a}$ is given by

$$
T_{a}(q, t)=T_{\infty}+\frac{q(t)}{4 \pi k}\left[\ln \left(\frac{4 \alpha t}{r_{b}^{2}}\right)-\gamma\right]-\frac{1}{4 \pi k} \int_{0}^{t} \frac{q(t)-q(\lambda)}{t-\lambda} \mathrm{d} \lambda .
$$

It can be seen that close to the borehole the outer solution behaves in a quasisteady way with an apparent temperature $T_{a}$ that depends on the history of the applied heat injection rate per unit borehole length $q(t)$. The emergence of an apparent temperature in the asymptotic matching process of outer and inner solutions is common when dealing with cylindrically or spherically symmetrical problems $[11,19,21]$. Since the outer and inner solutions must behave equally in the intermediate matching region, (3.13) has to be imposed as a boundary condition at infinity for the inner region instead of the original boundary condition given in (2.6):

$$
|\vec{x}| \rightarrow \infty: T \rightarrow-\frac{q(t)}{2 \pi k} \ln \left(\frac{r}{r_{b}}\right)+T_{a}(q, t) .
$$

4. Borehole thermal resistances. The quasi-steady zeroth order inner problem to be solved is comprised of the steady-state heat conduction equation (3.2), the boundary condition at the pipe walls $(2.1)$, the continuity conditions at the borehole wall (2.5), and the matching condition given in (3.15).

In the present section it is proven that, despite the more complex boundary condition to be imposed at the outer boundary of the inner region, the solution to the formulated problem can be expressed as a network of thermal resistances that depends only on the geometrical and thermal parameters of the problem, and that relates the heat injection rates per unit borehole length $q_{i}$ of each pipe $i$ with the temperature differences between each pipe and the apparent temperature $T_{a}$.

By convenient a priori grouping of variables and parameters and the subsequent application of the Pi-theorem from dimensional analysis [5], the following functional dependency of the nondimensional temperature on the independent variables and parameters of the problem is obtained:

$$
\frac{T-T_{a}}{q / k_{b}}=F\left(\frac{\vec{x}}{r_{b}}, \frac{T_{i}-T_{a}}{q / k_{b}} ; \frac{r_{i}}{r_{b}}, \frac{\vec{x}_{i}}{r_{b}}, k_{b} R_{p i}, \frac{k}{k_{b}}\right),
$$

where the nondimensional numbers that contain the subindex $i$ represent the complete set of nondimensional numbers for all $N$ pipes inside the borehole. This result can now be used to obtain the functional dependency of derived quantities, such as the heat injection rate per unit borehole length $q_{i}$ at each pipe $i$, defined as

$$
q_{i}=-\int_{0}^{2 \pi} k_{b} \frac{\partial T}{\partial n_{i}} r_{i} \mathrm{~d} \theta_{i}
$$

where $\theta_{i}$ is the azimuthal angle corresponding to the polar coordinate system centered in pipe $i$. Substitution of (4.1) into (4.2) leads to the following functional dependency for $q_{i}$ :

$$
\frac{q_{i}}{q}=F_{i}\left(\frac{T_{i}-T_{a}}{q / k_{b}} ; \frac{r_{i}}{r_{b}}, \frac{\vec{x}_{i}}{r_{b}}, k_{b} R_{p i}, \frac{k}{k_{b}}\right) .
$$

Thanks to the linearity of the thermal problem to be solved, its solution can be expressed as superposition of a set of basic solutions. Following the same procedure 
as in [10], these basic solutions correspond to the simpler problems in which a heat injection rate per unit borehole length $q_{i}$ is applied to pipe $i$, while the heat injection rates per unit borehole length for all the remaining pipes are set equal to zero. For the sake of simplicity in the forthcoming discussion, the particular case of a borehole with only two pipes is presented, the generalization to the case of $N$ pipes being straightforward. The resulting system of equations for the basic solution 1, obtained for $q_{1} / q=1$ and $q_{2} / q=0$, would be

$$
\begin{aligned}
& 1=F_{1}\left(\frac{T_{1}-T_{a}}{q_{1} / k_{b}}, \frac{T_{2}-T_{a}}{q_{1} / k_{b}} ; \frac{r_{i}}{r_{b}}, \frac{\vec{x}_{i}}{r_{b}}, k_{b} R_{p i}, \frac{k}{k_{b}}\right), \\
& 0=F_{2}\left(\frac{T_{1}-T_{a}}{q_{1} / k_{b}}, \frac{T_{2}-T_{a}}{q_{1} / k_{b}} ; \frac{r_{i}}{r_{b}}, \frac{\vec{x}_{i}}{r_{b}}, k_{b} R_{p i}, \frac{k}{k_{b}}\right),
\end{aligned}
$$

where the fact that $q=q_{1}$ has been used to rewrite the right-hand side of the expressions. This system of two equations for the two unknowns $\left(T_{1}-T_{a}\right) /\left(q_{1} / k_{b}\right)$ and $\left(T_{2}-T_{a}\right) /\left(q_{1} / k_{b}\right)$ has the following unique solution:

$$
\begin{aligned}
& \frac{T_{1}-T_{a}}{q_{1} / k_{b}}=C_{1}\left(\frac{r_{i}}{r_{b}}, \frac{\vec{x}_{i}}{r_{b}}, k_{b} R_{p i}, \frac{k}{k_{b}}\right), \\
& \frac{T_{2}-T_{a}}{q_{1} / k_{b}}=C_{2}\left(\frac{r_{i}}{r_{b}}, \frac{\vec{x}_{i}}{r_{b}}, k_{b} R_{p i}, \frac{k}{k_{b}}\right) .
\end{aligned}
$$

Uniqueness can be assured through the uniqueness of the solution to the underlying steady-state heat transfer problem [18]. In the literature these constants $C_{i}$, divided by the thermal conductivity $k_{b}$ of the grout material, are commonly called thermal resistances $\hat{R}_{1 i}=C_{i} / k_{b}$. Thus introducing this nomenclature and rearranging the expressions, the following final result is obtained:

$$
T_{1}-T_{a}=\hat{R}_{11} q_{1}, \quad T_{2}-T_{a}=\hat{R}_{12} q_{1} .
$$

Analogously, the system of equations for the basic solution 2, obtained for the heat injection rates per unit borehole length $q_{1} / q=0$ and $q_{2} / q=1$, leads to

$$
T_{1}-T_{a}=\hat{R}_{21} q_{2}, \quad T_{2}-T_{a}=\hat{R}_{22} q_{2} .
$$

As pointed out in $[3,10,16]$, by means of Maxwell's reciprocal theorem it can be shown that $\hat{R}_{12}=\hat{R}_{21}$.

By superposition of the obtained basic solutions, the general solution to the zeroth order inner problem can be written as follows:

$$
\begin{aligned}
& T_{1}-T_{a}=\hat{R}_{11} q_{1}+\hat{R}_{12} q_{2}, \\
& T_{2}-T_{a}=\hat{R}_{12} q_{1}+\hat{R}_{22} q_{2},
\end{aligned}
$$

showing that it can be represented as a network of thermal resistances that relates the heat injection rates per unit borehole length $q_{i}$ with the temperature differences between each pipe and the apparent temperature $T_{a}$. From (4.5) it can be seen that these thermal resistances depend only on the geometrical and thermal parameters of the problem. Two important notes must be made here. First, this result is exact and not only an approximation or model for the zeroth order inner problem. Therefore, relations between heat injection rates per unit borehole length and temperatures with 
a structure that differs from the one given in (4.8) are not consistent with the underlying physics. Second, the constants $C_{i}$ can attain negative values. This fact may lead to erroneous conclusions regarding the validity of the derived expressions, like in [22], when these constants are strictly reinterpreted as thermal resistances, as the latter ones are assumed to always be positive.

Solving the above system of equations for the heat injection rates per unit borehole length leads to

$$
\begin{aligned}
& q_{1}=\frac{T_{1}-T_{a}}{R_{1}}+\frac{T_{1}-T_{2}}{R_{12}}, \\
& q_{2}=\frac{T_{2}-T_{a}}{R_{2}}+\frac{T_{2}-T_{1}}{R_{12}},
\end{aligned}
$$

where

$$
\begin{gathered}
\frac{1}{R_{1}}=\frac{\hat{R}_{22}-\hat{R}_{12}}{\hat{R}_{11} \hat{R}_{22}-\hat{R}_{12}^{2}}, \quad \frac{1}{R_{2}}=\frac{\hat{R}_{11}-\hat{R}_{12}}{\hat{R}_{11} \hat{R}_{22}-\hat{R}_{12}^{2}}, \\
\frac{1}{R_{12}}=\frac{\hat{R}_{12}}{\hat{R}_{11} \hat{R}_{22}-\hat{R}_{12}^{2}} .
\end{gathered}
$$

A more convenient way of writing the above relationships is as follows:

$$
\begin{aligned}
& q_{1}=\frac{T_{m}-T_{a}}{R_{1}}+\frac{T_{1}-T_{2}}{R_{a}}, \\
& q_{2}=\frac{T_{m}-T_{a}}{R_{2}}+\frac{T_{2}-T_{1}}{R_{a}},
\end{aligned}
$$

where the internal thermal resistance between the two pipes $R_{a}$ is given by

$$
\frac{1}{R_{a}}=\frac{1}{R_{1}+R_{2}}+\frac{1}{R_{12}}
$$

and the following weighted mean fluid temperature $T_{m}$ is introduced:

$$
T_{m}=\frac{\frac{T_{1}}{R_{1}}+\frac{T_{2}}{R_{2}}}{\frac{1}{R_{1}}+\frac{1}{R_{2}}} .
$$

This weighted mean fluid temperature, which differs from the arithmetic mean fluid temperature $\left(T_{1}+T_{2}\right) / 2$ for geothermal boreholes that are not thermally symmetric $\left(R_{1} \neq R_{2}\right)$, is the temperature with which the borehole exchanges heat with the surrounding ground. To show it, the heat injection rate per unit borehole length $q=q_{1}+q_{2}$ is computed:

$$
q=\frac{T_{m}-T_{a}}{R_{b}}
$$

where the borehole thermal resistance $R_{b}$ is given by

$$
\frac{1}{R_{b}}=\frac{1}{R_{1}}+\frac{1}{R_{2}}
$$

Having reached this point, the still unknown heat injection rate per unit borehole length $q(t)$ can finally be obtained. By combining (4.14) with the expression for the 
apparent temperature $T_{a}$ given in (3.14), the following integro-algebraic equation for $q(t)$ is obtained:

$$
T_{m}(t)-T_{\infty}=R_{b} q(t)+\frac{q(t)}{4 \pi k}\left[\ln \left(\frac{4 \alpha t}{r_{b}^{2}}\right)-\gamma\right]-\frac{1}{4 \pi k} \int_{0}^{t} \frac{q(t)-q(\lambda)}{t-\lambda} \mathrm{d} \lambda .
$$

Although in general this equation has to be solved numerically, that is quite straightforward to do once a piecewise constant or linear approximation for $T_{m}(t)$ and $q(t)$ is considered, which is the usual approach followed in the analysis of geothermal boreholes $[4,13]$.

5. Existing models for thermal resistances. The considered limit of slowly varying heat injection rates has been extensively studied in the literature, and many software simulation packages for geothermal heat exchangers rely on it $[4,12,15,27$, 32 . Different approximate and exact approaches and models exist for the definition and computation of the thermal resistances that represent the quasi-steady solution to the inner region. Of interest for the present analysis are those that explicitly compute the thermal resistances out of the thermal problem defined in the two-dimensional plane perpendicular to the borehole. Therefore, all those methods that fit or infer thermal resistances out of experimental data, like in [28, 30], are not considered here.

In the present section a review of the most relevant and/or extended approaches and models is presented, and their merits and drawbacks are briefly discussed in view of the results derived in the present work. In this sense, it is worth noting that the definition of thermal resistance given in the present work, based on the zeroth order asymptotic matching of the outer and inner regions, is the formally correct one in the limit of slowly varying heat injection rates.

The first relevant work on borehole thermal resistances was done by researchers at Lund University $[3,9]$. Their approach was based on the analytical solution of the quasi-steady thermal problem in the inner region by means of a multipole expansion of the temperature field. The series was then truncated at a certain point to allow for the numerical solution of the system of algebraic equations resulting from the imposition of the boundary conditions of the thermal problem. Far from the borehole, a mixedtype boundary condition was imposed at a distance $r_{c}$ from the borehole center,

$$
r=r_{c}: T+2 \pi k R_{c} r_{c} \frac{\partial T}{\partial r}=T_{c},
$$

allowing for the inclusion of possible thermal contact resistances $R_{c}$ at that location. In the context of geothermal boreholes and the computation of the associated thermal resistances, a drawback of the proposed procedure is that the distance $r_{c}$ as well as the mixed-type boundary condition itself are arbitrary and not motivated by the underlying physical problem. Therefore, the dependence of the solution on them is unphysical. However, despite all that, it is possible to reinterpret the above mixed-type boundary condition as a numerical approximation at $r=r_{c}$ of the correct asymptotic matching condition given in (3.15) through the following suitable choice of the values of $R_{c}$ and $T_{c}$ :

$$
R_{c}=-\frac{1}{2 \pi k} \ln \left(\frac{r_{c}}{r_{b}}\right), \quad T_{c}=T_{a}
$$

Later on, Hellström [16] reviewed the multipole method and studied the behavior of the multipole expansion, and therefore of the temperature distribution, far from 
the borehole. He saw that the thermal resistances $\hat{R}_{m n, c}$ between pipes $m$ and $n$ behaved as follows when $r_{c} \gg r_{b}$ :

$$
\hat{R}_{m n, c}=\hat{R}_{m n}+\frac{1}{2 \pi k} \ln \left(\frac{r_{c}}{r_{b}}\right),
$$

where $\hat{R}_{m n}$ would not depend on $r_{c}$. Therefore, he introduced a new temperature $T_{b}$ at the borehole wall located at $r=r_{b}$, defined by

$$
T_{b}=T_{c}+\frac{q}{2 \pi k} \ln \left(\frac{r_{c}}{r_{b}}\right) .
$$

The advantage of this procedure is that the thermal resistances based on it, namely $\hat{R}_{m n}$, still account for the effect of the ground region outside of the borehole, and in principle do not depend on the specific value of $r_{c}$. But as shown by Hellström, the particular choice of $r_{c}$ still alters the numerical value of $\hat{R}_{m n}$, since an azimuthally constant temperature $T_{c}$ is imposed at $r_{c}$, and there is no physical reason for that. However, the influence of $r_{c}$ becomes negligible once $r_{c}$ is sufficiently large compared to $r_{b}[16]$.

The introduction of the above temperature $T_{b}$, which is by definition constant along the borehole wall, spawned the wrong impression that the correct way of computing thermal resistances was by solving the quasi-steady heat transfer problem strictly inside the borehole, and imposing $T_{b}$ as a Dirichlet boundary condition at the borehole wall. Good examples of such approaches can be found in [24, 31]. This is clearly wrong, as there is no physical justification for forcing the temperature to be constant there, leading to significant errors especially when the pipes are located close to the borehole wall, as shown in [22] and in the next section.

In their recent review, Lamarche, Kajl, and Beauchamp [22] compared several of the above-mentioned approaches to compute the thermal resistances with values derived from the long term evolution of detailed unsteady numerical simulations, and highlighted the flaws of imposing $T_{b}$ as a Dirichlet boundary condition at the borehole wall. They showed that the temperature varies considerably along the borehole wall, and they therefore proposed an alternative model based on the imposition of two different temperatures, each one constant over half of the borehole wall. Although their model seems to improve the previous one, it raises two important questions. First, the zeroth order outer solution returns only one temperature and not two. This a priori seems to render the coupling to the outer solution impossible. And second, the structure of the network of thermal resistances differs from that presented in (4.8); thus it should be either physically inconsistent or mathematically equivalent to (4.8).

It was not until very recently that the mathematically correct approach for computing thermal resistances was presented. Claesson and Hellström [10] reformulated the multipole expansion technique in order to completely eliminate the need for the outer circle located at $r_{c}$. Instead, they directly imposed that the mean azimuthal temperature at the borehole wall be equal to $T_{b}$, and not the temperature itself as in $[24,31]$. In this way, they derived a formulation for the thermal resistances that turns out to be equivalent to the one derived in the present work, as the boundary condition given in (3.15) leads to the same multipole expansion as their boundary condition on the mean azimuthal temperature.

Regarding the temperature $T_{b}$ to be imposed, Eskilson [13] took the value attained by the axisymmetric zeroth order outer solution at $r=r_{b}$. In the present case, in 
which the outer solution is given by (3.7), that would be

$$
T_{b}=T_{\infty}+\frac{1}{4 \pi k} \int_{0}^{t} \frac{q(\lambda)}{t-\lambda} \exp \left[-\frac{r_{b}^{2}}{4 \alpha(t-\lambda)}\right] \mathrm{d} \lambda .
$$

This same approach has extensively been used since then $[4,13,15,16,24,27,32]$. This choice of temperature differs from the apparent temperature $T_{a}$ derived in the present work, and that shall be used instead. However, it will be shown in the next section that the differences are small.

6. Comparison with existing models. The developed asymptotic solution is compared in the present section with the existing thermal models just presented as well as with exact solutions obtained by analytical or numerical integration of the thermal problem formulated in section 2. Three key aspects are analyzed: first, the errors introduced by the imposition of the Dirichlet boundary condition at the borehole wall instead of the asymptotic matching condition at infinity; second, the range of validity of the derived asymptotic solution; and third, the errors or differences between using the mean borehole temperature $T_{b}$, obtained by evaluating the outer solution at $r=r_{b}$, instead of the apparent temperature $T_{a}$.

6.1. Asymptotic condition versus Dirichlet condition. The first aspect to be analyzed is the errors introduced due to the computation of the borehole thermal resistance $R_{b}$ by imposing the Dirichlet boundary condition at $r=r_{b}$ instead of the asymptotic matching condition given in (3.15). When using the asymptotic matching condition, it can be concluded from section 4 that the nondimensional borehole thermal resistance $k_{b} R_{b}$ depends on the following nondimensional parameters:

$$
k_{b} R_{b}=f\left(\frac{r_{i}}{r_{b}}, \frac{\vec{x}_{i}}{r_{b}}, k_{b} R_{p i}, \frac{k}{k_{b}}\right) .
$$

To get an overall picture, the full range of reasonable values for the nondimensional parameters is considered here, limiting the study, however, for the sake of simplicity, to thermally symmetric single U-pipe borehole configurations with pipe and borehole diameters fixed, respectively, to the typical values of $32 \mathrm{~mm}$ and $152 \mathrm{~mm}$. The resulting nondimensional borehole thermal resistances $k_{b} R_{b}$ are obtained from the computation of the basic solutions introduced in section 4 . For this calculation, the zeroth order inner problem comprising the steady-state heat conduction equation (3.2), the boundary condition at the pipe walls (2.1), the continuity conditions at the borehole wall (2.5), and the matching condition given in (3.15) is solved using the multipole expansion technique presented in $[3,9,10]$. Figure 2 shows the resulting nondimensional borehole thermal resistances $k_{b} R_{b}$ as functions of the nondimensional distance $x_{p} / r_{b}$ of the pipe centers to the borehole center, the nondimensional ratio of thermal conductivities $k / k_{b}$, and the nondimensional inner thermal resistance $k_{b} R_{p}$ of the pipes. Also represented in the figure are the values for $k_{b} R_{b}$ obtained through the imposition of the Dirichlet boundary condition at the borehole wall instead of the asymptotic matching condition at infinity.

As expected, the results show that the values of the borehole thermal resistances drop as the pipes are moved towards the borehole wall. Unexpected is the fact that a minima exists for pipe locations close to the borehole wall when $k / k_{b}$ is small. However, the differences between the values attained at the minima and at the extreme location $x_{p} / r_{b}=1-r_{p} / r_{b}$ are negligible. 


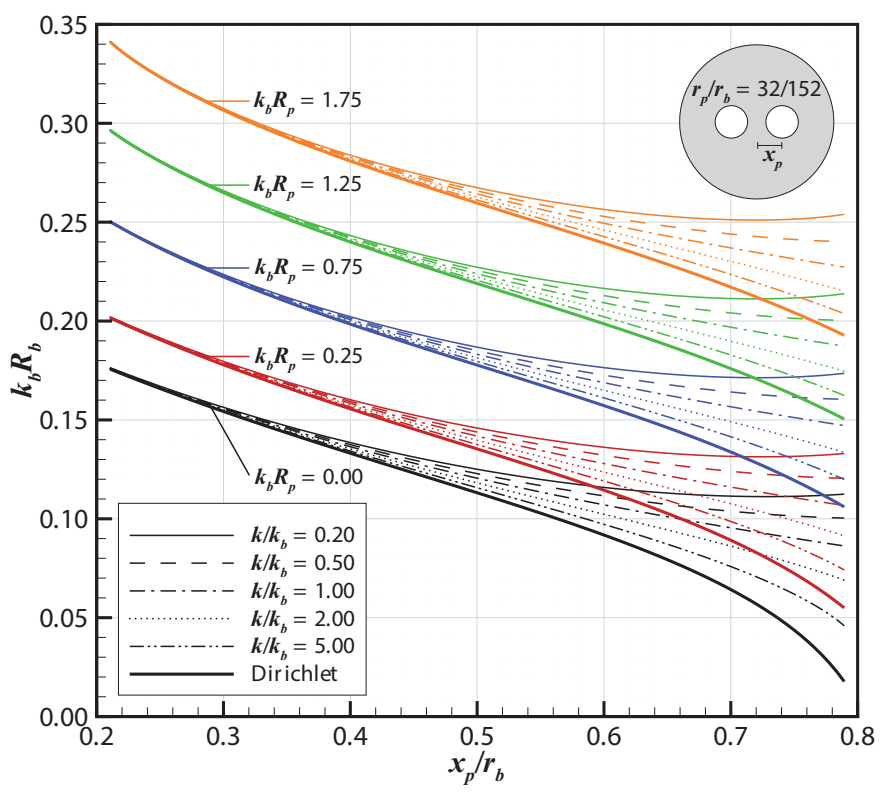

FIG. 2. Nondimensional borehole thermal resistance $k_{b} R_{b}$ for a thermally symmetric single $U$-pipe borehole configuration with pipe and borehole diameters equal to $32 \mathrm{~mm}$ and $152 \mathrm{~mm}$, respectively, as a function of the nondimensional distance $x_{p} / r_{b}$ of the pipe centers to the borehole center, the nondimensional ratio of thermal conductivities $k / k_{b}$, and the nondimensional inner thermal resistance $k_{b} R_{p}$ of the pipes. Also represented, with thicker lines, are the values obtained through the imposition of the Dirichlet boundary condition at the borehole wall instead of the asymptotic matching condition at infinity.

When comparing the borehole thermal resistances computed using the Dirichlet boundary condition with those computed using the asymptotic matching condition given in (3.15), the following three conclusions can be derived. First, since in the former case the problem to be solved does not take into account the surrounding ground, the obtained nondimensional borehole thermal resistances do not depend on the ratio of thermal conductivities $k / k_{b}$, leading to a single curve for each value of the nondimensional inner thermal resistance $k_{b} R_{p}$. Second, as the pipes are moved towards the borehole wall, the errors committed by the former approach increase, especially when $k / k_{b}$ is small, leading in some cases to errors well above $100 \%$. And third, the borehole thermal resistances predicted by the former approach are not conservative, meaning that borehole heat exchangers designed with them will have fewer heat exchange capabilities than predicted. This is due to the fact that the thermal borehole resistances computed using the Dirichlet boundary condition are always smaller than the correct ones, as seen in Figure 2.

6.2. Range of validity of the asymptotic solution. The next aspect to be analyzed is the range of validity of the derived asymptotic description of the solution in terms of characteristic times of variation $t_{q}$ of the heat injection rate. It is important to remember that the performed asymptotic analysis is based on the limit $t_{b} / t_{q} \ll 1$, and therefore for not so slowly varying heat injection rates the derived expressions are expected to become less accurate.

A time periodic problem is considered in all comparisons to be performed in the present and the next subsections, in which the fluid temperatures vary as

$$
T_{i}=T_{\infty}+\operatorname{Re}\left[\hat{T}_{i} \exp (i \omega t)\right],
$$


where $2 \pi / \omega$ is the time period of the harmonic variation of all the fluid temperatures, $\hat{T}_{i}$ the complex harmonic amplitude of $T_{i}$, and $i^{2}=-1$. In this way, analytical expressions are derived, and the spectral responses of the different methods can easily be compared. All the remaining variables of the problem vary in the same way, with the corresponding complex harmonic amplitudes denoted by a hat on top of the variables, like in $\hat{T}_{i}$.

By substituting the harmonic behaviors of the variables into (4.16), the following expression for the asymptotic solution is obtained:

$$
\begin{aligned}
\hat{T}_{m}=\lim _{t_{0} \rightarrow-\infty} \frac{\hat{q}}{4 \pi k} & {\left[4 \pi k R_{b}+\ln \left(\frac{4 \alpha\left(t-t_{0}\right)}{r_{b}^{2}}\right)-\gamma\right.} \\
& \left.-\int_{t_{0}}^{t} \frac{1-\exp [-i \omega(t-\lambda)]}{t-\lambda} \mathrm{d} \lambda\right],
\end{aligned}
$$

where it is necessary to change the initial condition from $t=0$ to $t=t_{0} \rightarrow-\infty$ because of the time periodicity of the problem and the absence of an initial condition. Evaluating the integral on the right-hand side and solving for the heat injection rate per unit borehole length leads to

$$
\frac{\hat{q}}{2 \pi k \hat{T}_{m}}=\left[2 \pi k R_{b}+\ln \left(\sqrt{4 \frac{\omega^{-1}}{r_{b}^{2} / \alpha}}\right)-\gamma-\frac{\pi}{4} i\right]^{-1} .
$$

The derived expression represents the spectral transfer function of the system and depends on the nondimensional borehole thermal resistance $k R_{b}=\left(k / k_{b}\right) k_{b} R_{b}$ and the ratio between the characteristic time of variation $t_{q} \sim \omega^{-1}$ and the characteristic transverse diffusion time $t_{b} \sim r_{b}^{2} / \alpha$.

A pipe-in-pipe coaxial borehole configuration, like the one depicted in Figure 3, is considered here to assess the range of validity of the above asymptotic expression. The simple geometrical configuration allows the analytical determination of the borehole thermal resistance following the steps outlined in section 4 , leading to

$$
k_{b} R_{b}=\frac{k_{b} R_{p}-\ln \left(r_{p} / r_{b}\right)}{2 \pi} .
$$

Additionally, analytical expressions for the exact time periodic thermal response of the pipe-in-pipe coaxial borehole configuration can be obtained, leading to the following expression for the nondimensional heat injection rate per unit borehole length:

$$
\frac{\hat{q}}{2 \pi k \hat{T}_{m}}=\frac{1}{K} \frac{A_{1} B_{1}-A_{2} B_{2}}{C_{1} B_{1}-C_{2} B_{2}}
$$

where

$$
\begin{aligned}
& A_{1}=-I_{1}(\sqrt{i \xi A} R) \sqrt{i \xi A} R \\
& A_{2}=K_{1}(\sqrt{i \xi A} R) \sqrt{i \xi A} R \\
& B_{1}=K_{0}(\sqrt{i \xi A}) K_{1}(\sqrt{i \xi}) K-K_{1}(\sqrt{i \xi A}) K_{0}(\sqrt{i \xi}) \sqrt{A} \\
& B_{2}=I_{0}(\sqrt{i \xi A}) K_{1}(\sqrt{i \xi}) K+I_{1}(\sqrt{i \xi A}) K_{0}(\sqrt{i \xi}) \sqrt{A} \\
& C_{1}=I_{0}(\sqrt{i \xi A} R)-I_{1}(\sqrt{i \xi A} R) \beta \sqrt{i \xi A} R \\
& C_{2}=K_{0}(\sqrt{i \xi A} R)+K_{1}(\sqrt{i \xi A} R) \beta \sqrt{i \xi A} R
\end{aligned}
$$




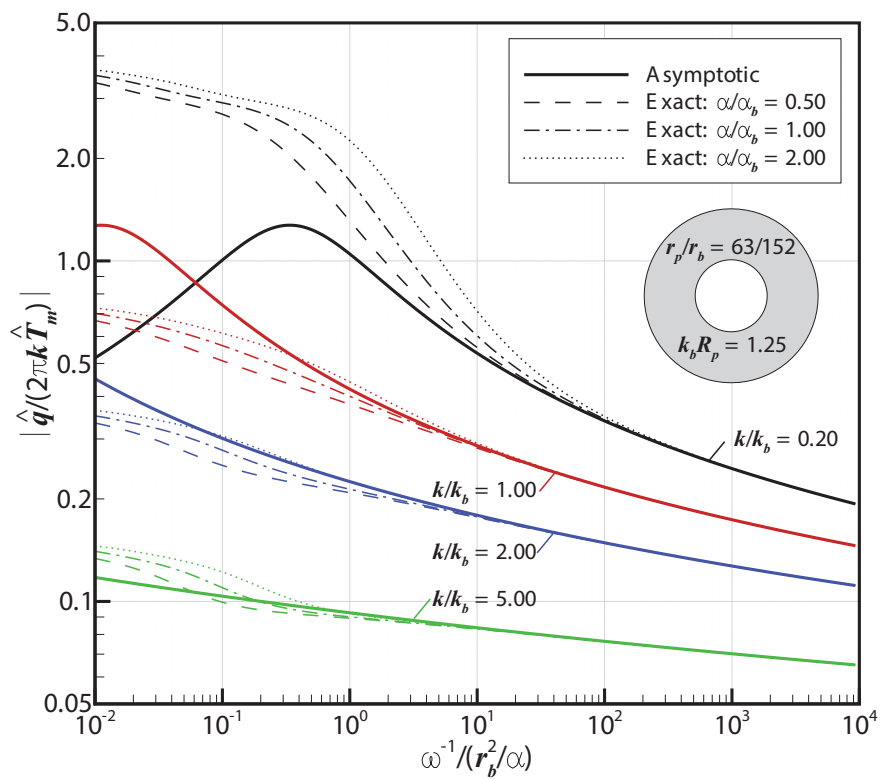

(a)

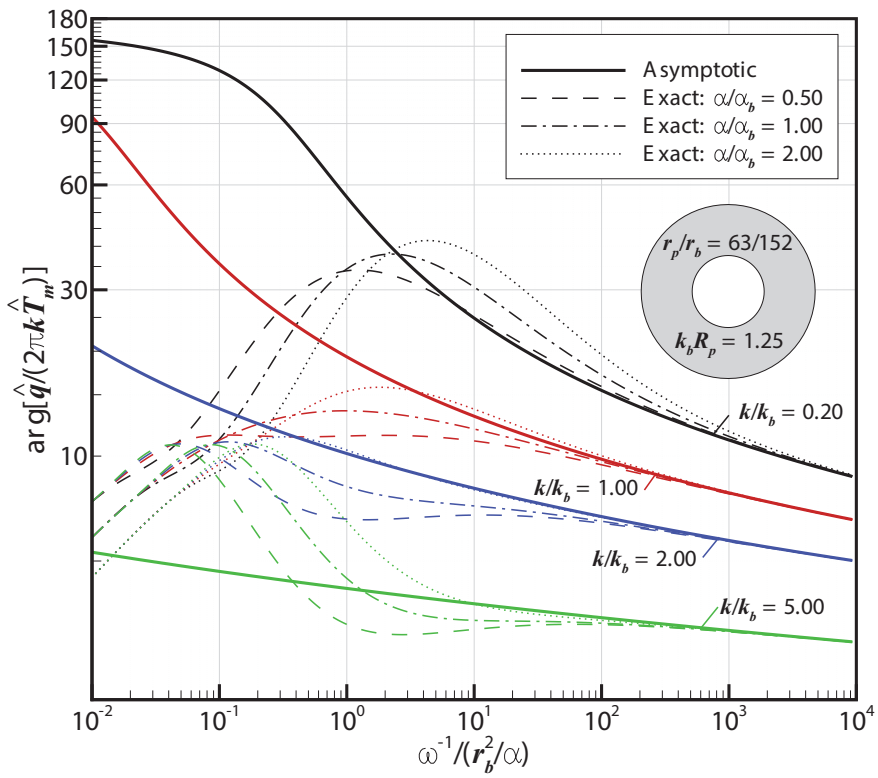

(b)

FIG. 3. (a) Modulus and (b) argument (in degrees) of the nondimensional heat injection rate per unit borehole length $\hat{q} /\left(2 \pi k \hat{T}_{m}\right)$ for a pipe-in-pipe coaxial borehole configuration with pipe and borehole diameters equal to $63 \mathrm{~mm}$ and $152 \mathrm{~mm}$, respectively, and a nondimensional inner thermal resistance of the pipes of $k_{b} R_{p}=1.25$ as functions of the ratio between the characteristic time of variation $\omega^{-1}$ of the mean fluid temperature $T_{m}$ and the characteristic transverse diffusion time $r_{b}^{2} / \alpha$ of the borehole and for different values of the ratio of thermal conductivities $k / k_{b}$. Shown are the values corresponding to the asymptotic solution (continuous lines) and the exact analytical solutions for different values of the ratio of thermal diffusivities $\alpha / \alpha_{b}$ (discontinuous lines). 
where $I_{n}(z)$ and $K_{n}(z)$ are the modified Bessel functions of the first and second kind, respectively, of order $n$, and

$$
\xi=\frac{r_{b}^{2} / \alpha}{\omega^{-1}}, \quad R=\frac{r_{p}}{r_{b}}, \quad K=\frac{k}{k_{b}}, \quad A=\frac{\alpha}{\alpha_{b}}, \quad \beta=k_{b} R_{p} .
$$

The exact thermal response of the borehole depends on an additional nondimensional parameter, namely the ratio of thermal diffusivities $\alpha / \alpha_{b}$. In contrast, the derived asymptotic solution does not depend on this ratio since in the first order asymptotic approximation the unsteady terms in the heat conduction equations are negligible close to the borehole, as shown in section 3 .

Figure 3 represents the modulus and argument of the nondimensional heat injection rate per unit borehole length $\hat{q} /\left(2 \pi k \hat{T}_{m}\right)$, as given by the asymptotic solution and by the exact analytical solution, as functions of the ratio between the characteristic time of variation $\omega^{-1}$ of the mean fluid temperature $T_{m}$ and the characteristic transverse diffusion time $r_{b}^{2} / \alpha$ of the borehole. The full range of reasonable values for the ratio of thermal conductivities $k / k_{b}$ and for the ratio of thermal diffusivities $\alpha / \alpha_{b}$ is considered here, while for the sake of simplicity the diameters of the coaxial pipe-in-pipe and of the borehole are fixed respectively to the typical values of $63 \mathrm{~mm}$ and $152 \mathrm{~mm}$, and the inner thermal resistance is set to $k_{b} R_{p}=1.25$.

Consistent with the presented analysis and results, the influence of the ratio of thermal diffusivities $\alpha / \alpha_{b}$ becomes negligible once the ratio of characteristic times is large enough. On the other hand, when the ratio of characteristic times is small enough, the influence of the ratio of thermal conductivities $k / k_{b}$ becomes negligible. This is due to the fact that the characteristic thermal penetration length associated with the characteristic time $\omega^{-1}$, of order $\sqrt{\alpha / \omega}$, is smaller than the radius of the borehole, meaning that the surrounding ground is not reached by the imposed periodic temperature variation and therefore its thermal conductivity does not play a role in the thermal response of the borehole. This can be seen best in Figure 3(b), where for small values of $\omega^{-1} /\left(r_{b}^{2} / \alpha\right)$ the curves corresponding to different values of $k / k_{b}$ collapse into a single curve for each value of $\alpha / \alpha_{b}$.

As expected, the asymptotic approximation departs from the exact solution as the ratio of characteristic times $\omega^{-1} /\left(r_{b}^{2} / \alpha\right)$ becomes smaller and the hypothesis of slowly varying heat injection rate ceases to be valid, with the differences smaller in the modulus than in the argument of $\hat{q} /\left(2 \pi k \hat{T}_{m}\right)$. In general, the asymptotic approximation can be considered sufficiently accurate for ratios of characteristic times on the order of $10^{2}$ and above, although for highly conducting grounds sufficiently accurate results are already obtained for ratios on the order of $10^{1}$.

6.3. Apparent temperature versus outer solution at $r=r_{b}$. The third aspect to be analyzed is the differences that arise due to the use of the outer solution evaluated at $r=r_{b}$ instead of the apparent temperature $T_{a}$ defined in (3.14). To derive the spectral transfer function for the former approach, first the harmonic behaviors of the variables are substituted into (5.5), leading to

$$
\begin{aligned}
\hat{T}_{b} & =\lim _{t_{0} \rightarrow-\infty} \frac{\hat{q}}{4 \pi k} \int_{t_{0}}^{t} \frac{\exp [-i \omega(t-\lambda)]}{t-\lambda} \exp \left[-\frac{r_{b}^{2} / \alpha}{4(t-\lambda)}\right] \mathrm{d} \lambda \\
& =\frac{\hat{q}}{4 \pi k} 2 K_{0}\left(\sqrt{i \frac{r_{b}^{2} / \alpha}{\omega^{-1}}}\right),
\end{aligned}
$$

where again it is necessary to change the initial condition from $t=0$ to $t=t_{0} \rightarrow-\infty$ because of the time periodicity of the problem and the absence of an initial condition. 
Afterwards, this result is used to substitute the apparent temperature $T_{a}$ in (4.14). Solving then for the heat injection rate per unit borehole length leads to the desired spectral transfer function, which depends on the same nondimensional parameters as the spectral transfer function of the asymptotic solution:

$$
\frac{\hat{q}}{2 \pi k \hat{T}_{m}}=\left[2 \pi k R_{b}+K_{0}\left(\sqrt{i \frac{r_{b}^{2} / \alpha}{\omega^{-1}}}\right)\right]^{-1} .
$$

The comparison between this expression and the asymptotic one given in (6.4) is carried out for the thermally symmetric single U-pipe borehole configuration introduced in subsection 6.1. From the four nondimensional parameters that alter the value of the nondimensional borehole thermal resistance, only $k / k_{b}$ is varied in order to see the effect of different values of $k R_{b}$ on the temporal response of the solutions, while all the remaining parameters are kept fixed at

$$
\frac{r_{p}}{r_{b}}=\frac{32}{152}, \quad \frac{x_{p}}{r_{b}}=0.70, \quad k_{b} R_{p}=1.25 .
$$

The computed nondimensional borehole thermal resistances $k R_{b}$ are given in Table 6.1 for the four nondimensional ground thermal conductivities $k / k_{b}$ chosen for the present comparison and which cover the full range of reasonable values of the parameter.

TABLE 6.1

Values of the nondimensional ground thermal conductivity $k / k_{b}$ and of the corresponding nondimensional borehole thermal resistance $k R_{b}$ to be used in the comparison of the asymptotic expression, the expression obtained from imposing the outer solution at $r=r_{b}$, and the numerically obtained exact solution to the thermally symmetric single U-pipe borehole configuration.

\begin{tabular}{|c|cccc|}
\hline$k / k_{b}$ & 5.485 & 2.670 & 1.017 & 0.238 \\
\hline$k R_{b}$ & 1.000 & 0.500 & 0.200 & 0.050 \\
\hline
\end{tabular}

Both expressions are also compared with the exact solution obtained from solving the complete thermal problem formulated in section 2 with $T_{1}=T_{2}=T_{m}$. Instead of performing the numerical time integration of the problem, the harmonic behavior for the variables defined in (6.2) is substituted into the governing equations, leading to complex-valued Poisson equations that are then solved numerically. The spatial discretization of these equations is performed using an in-house developed first order finite volume method code based on Voronoi dual control volumes [20]. Figure 4 shows a coarsened version of one of the employed unstructured spatial meshes. The resulting system of complex algebraic equations is finally solved using the multifrontal massively parallel sparse direct solver MUMPS $[1,2]$.

Figure 5 represents the modulus and argument of the nondimensional heat injection rate per unit borehole length $\hat{q} /\left(2 \pi k \hat{T}_{m}\right)$, as given by the asymptotic expression, the expression obtained by imposing the outer solution at $r=r_{b}$, and by the numerically obtained exact solution, as functions of the ratio between the characteristic time of variation $\omega^{-1}$ of the mean fluid temperature $T_{m}$ and the characteristic transverse diffusion time $r_{b}^{2} / \alpha$ of the borehole. Different values of the nondimensional borehole thermal resistance $k R_{b}$ are considered, while the corresponding values for the nondimensional parameters fixed in previous paragraphs and in Table 6.1 are used for the numerically obtained exact solution. Since the exact thermal problem also depends on the ratio of thermal diffusivities $\alpha / \alpha_{b}$, three different values have been considered here, which cover the full range of reasonable values of the parameter. 


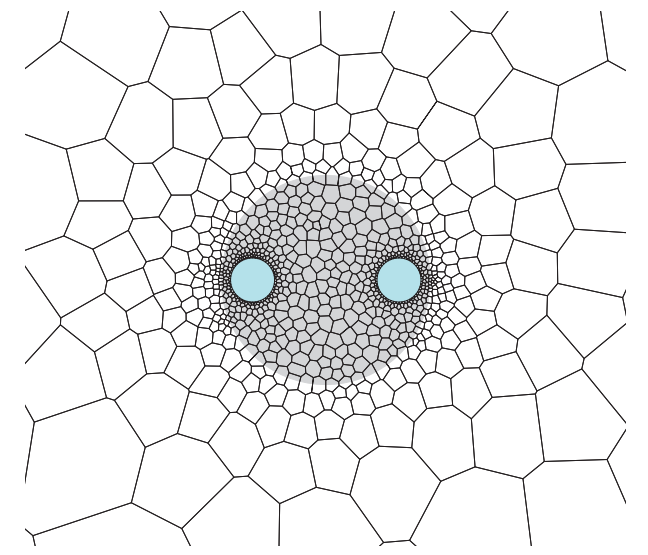

FIG. 4. Coarsened version of one of the unstructured meshes based on Voronoi dual control volumes employed in the numerical computation of the exact time periodic thermal response of single $U$-pipe borehole configurations by means of a first order finite volume discretization of the governing equations.

Conclusions can be derived from the present comparison similar to those from the previous one, namely, that the asymptotic approximation departs from the exact thermal response as the ratio of characteristic times $\omega^{-1} /\left(r_{b}^{2} / \alpha\right)$ becomes smaller. Again, sufficiently accurate results are obtained for ratios on the order of $10^{2}$ and above, although for highly conducting grounds sufficiently accurate rates are already obtained for ratios on the order of $10^{1}$. Comparing the asymptotic approximation with the one obtained from imposing the outer solution directly at $r=r_{b}$, it can be seen that both perform similarly in their range of validity. This result is not surprising, despite the latter not being formally correct, since the asymptotic matching condition derived in the present work is an integral part of the outer solution given by (3.7) and that is directly evaluated at $r=r_{b}$ in the latter approach.

7. Conclusions. In the present work the thermal response of vertical geothermal boreholes in the limit of slowly varying heat injection rates has been studied using matched asymptotic expansion techniques. The zeroth order approximation to the problem shows the existence of a quasi-steady region close to the borehole and an unsteady region far from the borehole. The asymptotic matching between these two regions delivers the correct outer boundary condition to be used in the computation of the network of thermal resistances that represents the quasi-steady solution to the inner region. The asymptotic matching also delivers an apparent temperature $T_{a}$ imposed by the outer solution onto the inner solution that is the one to be used when computing the heat injection rates per unit borehole length.

It was also shown that the above-mentioned network of thermal resistances must have a specific mathematical structure, as otherwise it is not consistent with the functional relationships derived from dimensional analysis. Additionally, we discussed that negative values for some of the thermal resistances are a physically correct result despite the fact that thermal resistances are normally assumed to be positive.

The presented comparison with existing models for thermal resistances shows that the approach based on the imposition of the outer solution as a Dirichlet boundary condition at the borehole wall leads to significantly underpredicted borehole thermal resistances, with errors in some cases well above $100 \%$. The consequence of this is that 


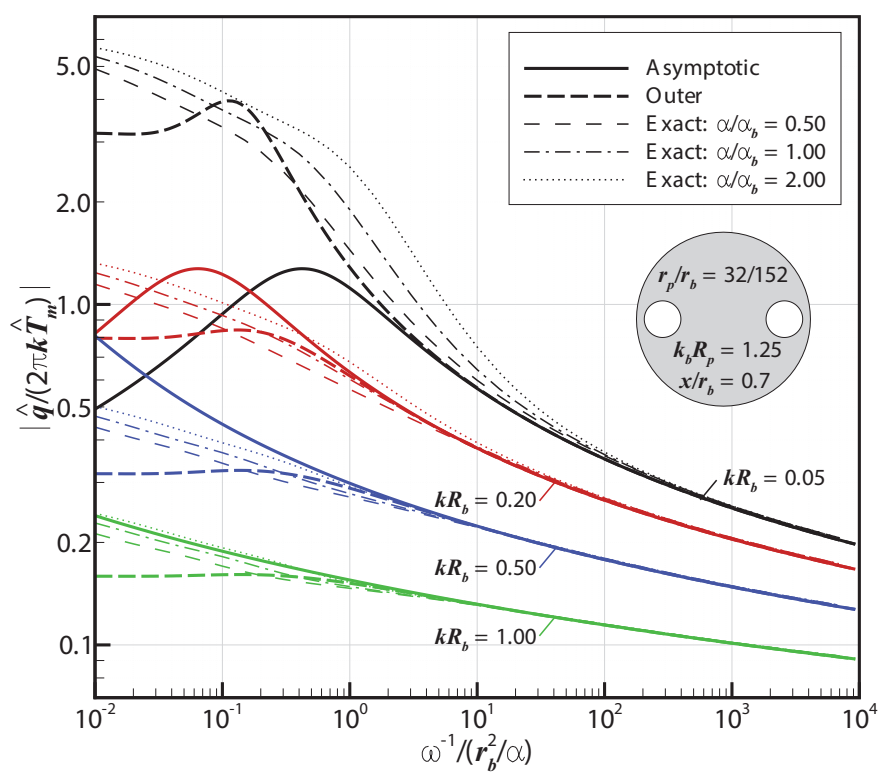

(a)

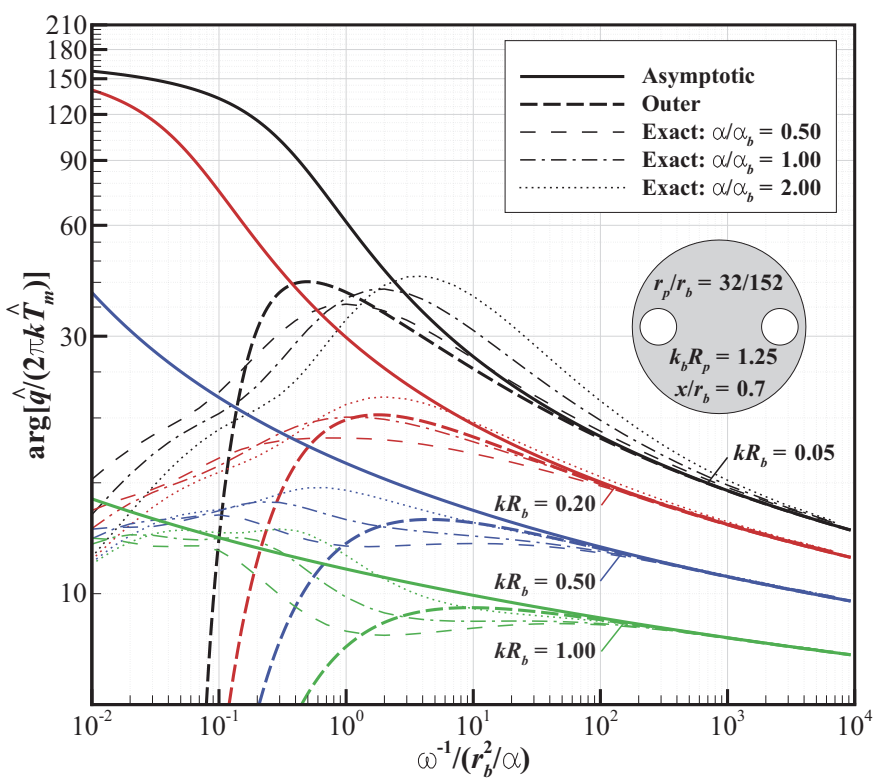

(b)

FIG. 5. (a) Modulus and (b) argument (in degrees) of the nondimensional heat injection rate per unit borehole length $\hat{q} /\left(2 \pi k \hat{T}_{m}\right)$ for a thermally symmetric single U-pipe borehole configuration with pipe and borehole diameters equal to $32 \mathrm{~mm}$ and $152 \mathrm{~mm}$, respectively, nondimensional pipe center distance to borehole center of $x / r_{b}=0.70$, and a nondimensional inner thermal resistance of the pipes of $k_{b} R_{p}=1.25$ as functions of the ratio between the characteristic time of variation $\omega^{-1}$ of the mean fluid temperature $T_{m}$ and the characteristic transverse diffusion time $r_{b}^{2} / \alpha$ of the borehole and for different values of the nondimensional borehole thermal resistance $k R_{b}$. Shown are the values corresponding to the asymptotic expression (thick continuous lines), the expression obtained from imposing the outer solution at $r=r_{b}$ (thick dashed lines), and the numerically obtained exact solution for different values of the ratio of thermal diffusivities $\alpha / \alpha_{b}$ (other discontinuous lines). 
geothermal heat exchangers designed with them will perform worse than predicted. In contrast, the theoretical comparison with the approach presented by Claesson and Hellström in [10] reveals that theirs is formally correct and therefore fully equivalent to the definition of thermal resistance given here.

The analysis of the range of validity of the zeroth order asymptotic approximation shows that sufficiently accurate results are obtained for ratios of the characteristic time of variation $\omega^{-1}$ of the mean fluid temperature $T_{m}$ and the characteristic transverse diffusion time $r_{b}^{2} / \alpha$ of the borehole on the order of $10^{2}$ and above, and that for highly conducting grounds sufficiently accurate results are already obtained for ratios on the order of $10^{1}$. Additionally, the comparison between the derived asymptotic analysis with its apparent temperature $T_{a}$ and the usual approach of directly using the outer solution evaluated at $r=r_{b}$ shows that both perform similarly in their range of validity despite the latter not being formally correct.

Thanks to the use of matched asymptotic expansion techniques, the present work sets the foundations for a more systematic approach to the analysis of the heat transfer problem in vertical geothermal boreholes. This approach allows the coherent integration of future improvements into the analysis. For example, inclusion of the next term in the asymptotic expansion not only introduces radial corrections to the presented zeroth order approximation, leading to an extended range of validity, but also the first azimuthal corrections that lead to unsteady thermal dipoles in the region far from the borehole. These dipoles would allow a better prediction of the thermal interference between adjacent vertical geothermal boreholes. Or, for example, the inclusion, through a multiscale variant of the present analysis, of short term variations in the heat injection rate, typically caused by the starting and stopping of geothermal heat pumps, would allow one to take into account changes of operation with characteristic times of variation on the order of the characteristic transverse diffusion time.

\section{REFERENCES}

[1] P.R. Amestoy, I.S. Duff, J.-Y. L'Excellent, And J. Koster, A fully asynchronous multifrontal solver using distributed dynamic scheduling, SIAM J. Matrix Anal. Appl., 23 (2001), pp. $15-41$.

[2] P.R. Amestoy, A. Guermouche, J.-Y. L'Excellent, and S. Pralet, Hybrid scheduling for the parallel solution of linear systems, Parallel Comput., 32 (2006), pp. 136-156.

[3] J. Bennet, J. Claesson, and G. Hellström, Multipole Method to Compute the Conductive Heat Flows to and between Pipes in a Composite Cylinder, Notes on Heat Transfer 3-1987, Department of Building Physics, Lund Institute of Technology, Lund, Sweden, 1987.

[4] T. Blomberg, J. Claesson, P. Eskilson, G. Hellström, and B. Sanner, Earth Energy Designer 3.0, User's Manual, Blocon Sweden, Sweden, 2008.

[5] P.W. Bridgman, Dimensional Analysis, Yale University Press, New Haven, CT, 1922.

[6] J. Busby, M. Lewis, H. Reeves, And R. LaWley, Initial geological considerations before installing ground source heat pump systems, Quart. J. Eng. Geol. Hydroge., 42 (2009), pp. 295-306.

[7] H.S. Carslaw and J.C. Jaeger, Conduction of Heat in Solids, 2nd ed., Oxford University Press, Oxford, UK, 1959.

[8] J.C. ChOI, S.R. LEE, AND D.S. LeE, Numerical simulation of vertical ground heat exchangers: Intermittent operation in unsaturated soil conditions, Comput. Geotech., 38 (2011), pp. 949-958.

[9] J. Claesson and J. Bennet, Multipole Method to Compute the Conductive Heat Flows to and between Pipes in a Cylinder, Notes on Heat Transfer 2-1987, Department of Building Physics, Lund Institute of Technology, Lund, Sweden, 1987.

[10] J. Claesson and G. Hellström, Multipole method to calculate borehole thermal resistances in a borehole heat exchanger, HVAC\&R Res., 17 (2011), pp. 895-911.

[11] A. Crespo And A. LiÑán, Unsteady effects in droplet evaporation and combustion, Combust. Sci. Technol., 11 (1975), pp. 9-18. 
[12] EnergyPlus Engineering Reference, software manual, Universities of Illinois and California, 2011.

[13] P. Eskilson, Thermal Analyses of Heat Extraction Boreholes, Ph.D. thesis, Department of Building Physics, Lund Institute of Technology, Lund, Sweden, 1987.

[14] O.T. FArouki, Thermal Properties of Soils, CRREL Monograph 81-1, U.S. Army Corps of Engineers, Cold Regions Research and Engineering Laboratory, Hanover, NH, 1981.

[15] GLHEPRO 4.0 for Windows: User's Guide, School of Mechanical and Aerospace Engineering, Oklahoma State University, Stillwater, OK, 2007.

[16] G. Hellström, Ground Heat Storage: Thermal Analyses of Duct Storage Systems, Ph.D. thesis, Department of Building Physics, Lund Institute of Technology, Lund, Sweden, 1991.

[17] International Energy Agency, Renewables for Heating and Cooling: Untapped Potential, Paris, France, 2007.

[18] F. John, Partial Differential Equations, 4th ed., Springer-Verlag, New York, 1981.

[19] S. Kaplun and P.A. Lagerstrom, Asymptotic expansions of Navier-Stokes solutions for small Reynolds numbers, J. Math. Mech., 6 (1957), pp. 585-593.

[20] P. Knabner and L. Angermann, Numerical Methods for Elliptic and Parabolic Partial Differential Equations, Springer, New York, 2003.

[21] P.A. Lagerstrom, Matched Asymptotic Expansions: Ideas and Techniques, Springer-Verlag, New York, 1988.

[22] L. Lamarche, S. Kajl, and B. Beauchamp, A review of methods to evaluate borehole thermal resistances in geothermal heat-pump systems, Geothermics, 39 (2010), pp. 187-200.

[23] Z. Li And M. Zheng, Development of a numerical model for the simulation of vertical U-tube ground heat exchangers, Appl. Therm. Eng., 29 (2009), pp. 920-924.

[24] K. Nagano, T. Katsura, and S. Takeda, Development of a design and performance prediction tool for the ground source heat pump system, Appl. Therm. Eng., 26 (2006), pp. $1578-1592$.

[25] Y. Nam, R. Ooka, And S. Hwang, Development of a numerical model to predict heat exchange rates for a ground-source heat pump system, Energ. Buildings, 40 (2008), pp. 2133-2140.

[26] P.H. Oosthuizen and D. NaYlor, An Introduction to Convective Heat Transfer Analysis, McGraw-Hill, New York, 1998.

[27] D. Pahud, A. Fromentin, And J.C. Hadorn, The Superposition Borehole Model for TRNSYS (TRNSBM), User's Manual for the November 1996 Version, Internal Report, Laboratoire des systèmes énergétiques, Déparement de Génie Civil, Ecole Polytechnique Fédérale de Lausanne, Lausanne, Switzerland, 1996.

[28] N.D. Paul, The Effect of Grout Thermal Conductivity on Vertical Geothermal Heat Exchanger Design and Performance, Master's thesis, South Dakota State University, Brookings, SD, 1996.

[29] E.C. Robertson, Thermal Properties of Rocks, United States Department of Interior, Geological Survey, Reston, VA, 1988.

[30] C.P. Remund, Borehole thermal resistance: Laboratory and field studies, ASHRAE Trans., 105 (1999), pp. 439-445.

[31] M.H. Sharqawy, E.M. Mokheimer, and H.M. Badr, Effective pipe-to-borehole thermal resistance for vertical ground heat exchangers, Geothermics, 38 (2009), pp. 271-277.

[32] M. Wetter And A. Huber, Vertical Borehole Heat Exchanger EWS Model, Version 2.4: Model Description and Implementing into TRNSYS, ZTL Lucern, Lucern, Switzerland, 1997.

[33] Y.S. XU AND D.D.L. Chung, Effect of sand addition on the specific heat and thermal conductivity of cement, Cem. Concr. Res., 30 (2000), pp. 59-61.

[34] Y. Xu AND D.D.L. Chung, Cement of high specific heat and high thermal conductivity, obtained by using silane and silica fume as admixtures, Cem. Concr. Res., 30 (2000), pp. 1175-1178.

[35] A. Zarrella, M. SCARPa, AND M. De CARli, Short time-step performances of coaxial and double U-tube borehole heat exchangers: Modeling and measurements, HVAC\&R Res., 17 (2011), pp. 959-976. 